\title{
Combined opioid free and loco-regional anaesthesia enhances the quality of recovery in sleeve gastrectomy done under ERAS protocol: a randomized controlled trial
}

\author{
Mohamed Ibrahim ${ }^{1,2^{*}}$, Ali M. Elnabtity ${ }^{1}$ Ahmed Hegab', Omar A. Alnujaidi² and Osama El Sanea²
}

\begin{abstract}
Background: It is debatable whether opioid-free anaesthesia (OFA) is better suited than multimodal analgesia (MMA) to achieve the goals of enhanced recovery after surgery (ERAS) in patients undergoing laparoscopic sleeve gastrectomy.

Methods: In all patients, anaesthesia was conducted with an i.v. induction with propofol (2 mg. kg-1), myorelaxation with cisatracurium $(0.15 \mathrm{mg} . \mathrm{kg}-1)$, in addition to an ultrasound-guided bilateral oblique subcostal transverse abdominis plane block. In addition, patients in the OFA group $(n=51)$ received i.v. dexmedetomidine $0.1 \mu \mathrm{g} . \mathrm{kg}-1$ and ketamine $(0.5 \mathrm{mg} . \mathrm{kg}-1)$ at induction, then dexmedetomidine $0.5 \mu \mathrm{g}$. kg-1.h-1, ketamine $0.5 \mathrm{mg} . \mathrm{kg}-1$.h-1, and lidocaine $1 \mathrm{mg}$. kg-1.h-1 for maintenance, while patients in the MMA group ( $n=52)$ had only i.v. fentanyl $(1 \mu \mathrm{\mu g}$. kg-1) at induction. The primary outcome was the quality of recovery assessed by QoR-40, at the 6th and the 24th postoperative hour. Secondary outcomes were postoperative opioid consumption, time to ambulate, time to tolerate oral fluid, and time to readiness for discharge.
\end{abstract}

Results: At the 6th hour, the QoR-40 was higher in the OFA than in the MMA group (respective median [IQR] values: 180 [173-195] vs. 185 [173-191], $p<0.0001$ ), but no longer difference was found at the 24th hour (median values $=191$ in both groups). OFA also significantly reduced postoperative pain and morphine consumption (20 mg [1-21] vs. $10 \mathrm{mg}$ [1-11], $p=0.005$ ), as well as time to oral fluid tolerance (238 [151-346] vs. $175 \mathrm{~min}$ [98-275], $p=0.022$ ), and readiness for discharge (505 [439-626] vs. $444 \mathrm{~min}$ [356-529], $p=0.001$ ), but did not influence time to ambulate.

Conclusion: While regional anaesthesia achieved most of the intraoperative analgesia, avoiding intraoperative opioids with the help of this OFA protocol was able to improve several sensible parameters of postoperative functional recovery, thus improving our knowledge on the OFA effects.

Clinical trial number: Registration number NCT04285255.

Keywords: Opioid free anaesthesia, Multimodal analgesia, Quality of recovery, Opioid consumption

*Correspondence: mibrahim72@hotmail.com

1 Department of Anesthesiology, Faculty of Medicine, Zagazig University,

Zagazig, Egypt

Full list of author information is available at the end of the article

(c) The Author(s) 2022. Open Access This article is licensed under a Creative Commons Attribution 4.0 International License, which permits use, sharing, adaptation, distribution and reproduction in any medium or format, as long as you give appropriate credit to the original author(s) and the source, provide a link to the Creative Commons licence, and indicate if changes were made. The images or other third party material in this article are included in the article's Creative Commons licence, unless indicated otherwise in a credit line to the material. If material is not included in the article's Creative Commons licence and your intended use is not permitted by statutory regulation or exceeds the permitted use, you will need to obtain permission directly from the copyright holder. To view a copy of this licence, visit http://creativecommons.org/licenses/by/4.0/. The Creative Commons Public Domain Dedication waiver (http://creativeco mmons.org/publicdomain/zero/1.0/) applies to the data made available in this article, unless otherwise stated in a credit line to the data. 


\section{Key points}

- Combined Opioid free and loco-regional anaesthesia provides enhanced early recovery

- Opioid free reduces postoperative pain intensity, and opioid consumption.

- Opioids free anesthesia allows early tolerance of oral fluid intake and expedites readiness for discharge than multimodal analgesia.

\section{Background}

Morbid obesity is the leading cause to premature death worldwide. Bariatric surgery remains the only proven effective and durable therapy thus far. However, safety concerns; poor functional outcome including food intolerance, pain and downtime represent the main deterrents for many patients to undergo bariatric surgery. The simplicity and success of laparoscopic sleeve gastrectomy have encouraged more patients to undergo bariatric surgery and the focus has shifted on improving acceptance of the procedure including shifting it to the ambulatory care setting $[1,2]$.

ERAS represents the best comprehensive quality improvement program offered with laparoscopic sleeve gastrectomy to improve accessibility to the procedure. While many anaesthetic considerations in ERAS have been well established including the multimodal analgesic approach, there is still debate as to whether opioid-free anaesthesia may offer additional benefit over multimodal analgesia to achieve the goals of ERAS [3].

Opioids increase nausea, vomiting, and the likelihood of respiratory depression in the peri-operative period, especially in morbidly obese patients [4]. Furthermore, opioids may delay patient meeting extubation criteria and cause hypoventilation, muscle fatigue, ileus, and urinary retention [5].

Opioid free anaesthesia limits opioid use during the peri-operative period through the use of loco-regional anaesthesia to block nociception [6]; and use of non opioid agents with proven analgesic efficacy like dexmedetomidine, an $\alpha 2$ agonist $[7,8]$, lidocaine $[9,10]$ or ketamine, a NMDA receptor blocker $[11,12]$. Several reviews compared opioid anaesthesia to opioid-free anaesthesia in the bariatric population but failed to provide reliable or valid conclusions due to lack of study power or variability in drug therapy or measured outcome $[13,14]$.

Our study, a single-blinded randomized controlled trial, compared the early postoperative quality of recovery between multimodal analgesia and opioid-free anaesthesia under ERAS in adult patients undergoing laparoscopic sleeve gastrectomy using the quality of recovery 40 (QoR-40) questionnaire at 6 and 24h [15]. Postoperative readiness to discharge, time to ambulate, and tolerate oral fluid, degree of pain control and subsequent opioid consumption were also studied as secondary outcomes.

\section{Methods}

The study was conducted at Almashfa Medical Center in Alkhobar Saudi Arabia between March and August 2020 (ClinicalTrials.gov: NCT04285255; registered Study Chair: Mohamed Ibrahim; registration date: February 2020). Approval was provided by Almashfa ethics committee (Chairperson Dr. Mohamed Ramadan) on January 20,2020 , under the number $1 / 1-2020$. The trial was registered before patient enrollment. A hundred and eight patients provided written informed consent to enroll in the trial before undergoing laparoscopic sleeve gastrectomy. This manuscript adheres to the applicable Equator guidelines.

\section{Study design}

A single-blinded randomized control trial was designed using the sealed envelope method and computer-generated random numbers were kept with a pharmacist. The original random allocation sequence was locked and a copy was used instead. A non-participant nurse anaesthetist prepared syringes with the study medications (propofol, ketamine, dexmedetomidine, lidocaine, and bupivacaine for oblique subcostal transversus abdominis plane (OSTAP) block and placed them into opaque envelopes according to the allocation orders (Fig. 1).

The patient, surgical team, operating theatre staff, PACU, and surgical ward nurses were blinded to both groups. The anesthesiologist or principal investigator was the only individual aware of patient allocation and administered anaesthesia to all study patients. Sealed envelopes were labelled as multimodal analgesia (MMA) or Opioid free anaesthesia (OFA) and envelopes were only opened upon patient entry into the operating theatre.

Eligible patients included adult patients between the age of 18 and 65; Body mass index between 40 and 60; American Society of anaesthesia (ASA) class II or III; planned for elective laparoscopic.

sleeve gastrectomy through trocars positioned at or above the umbilicus (T10 dermatome). Exclusion criteria included preoperative chronic use of opioid or NSAID; allergy to bupivacaine; local skin infection at the injection site of OSTAP (oblique subcostal transverse abdominis plane block); liver or renal insufficiency; psychiatric, or neurological disease; prior open abdominal surgery above T10 dermatome; patients converted to open surgery; and patients expected to be subjected to more tissue trauma. 


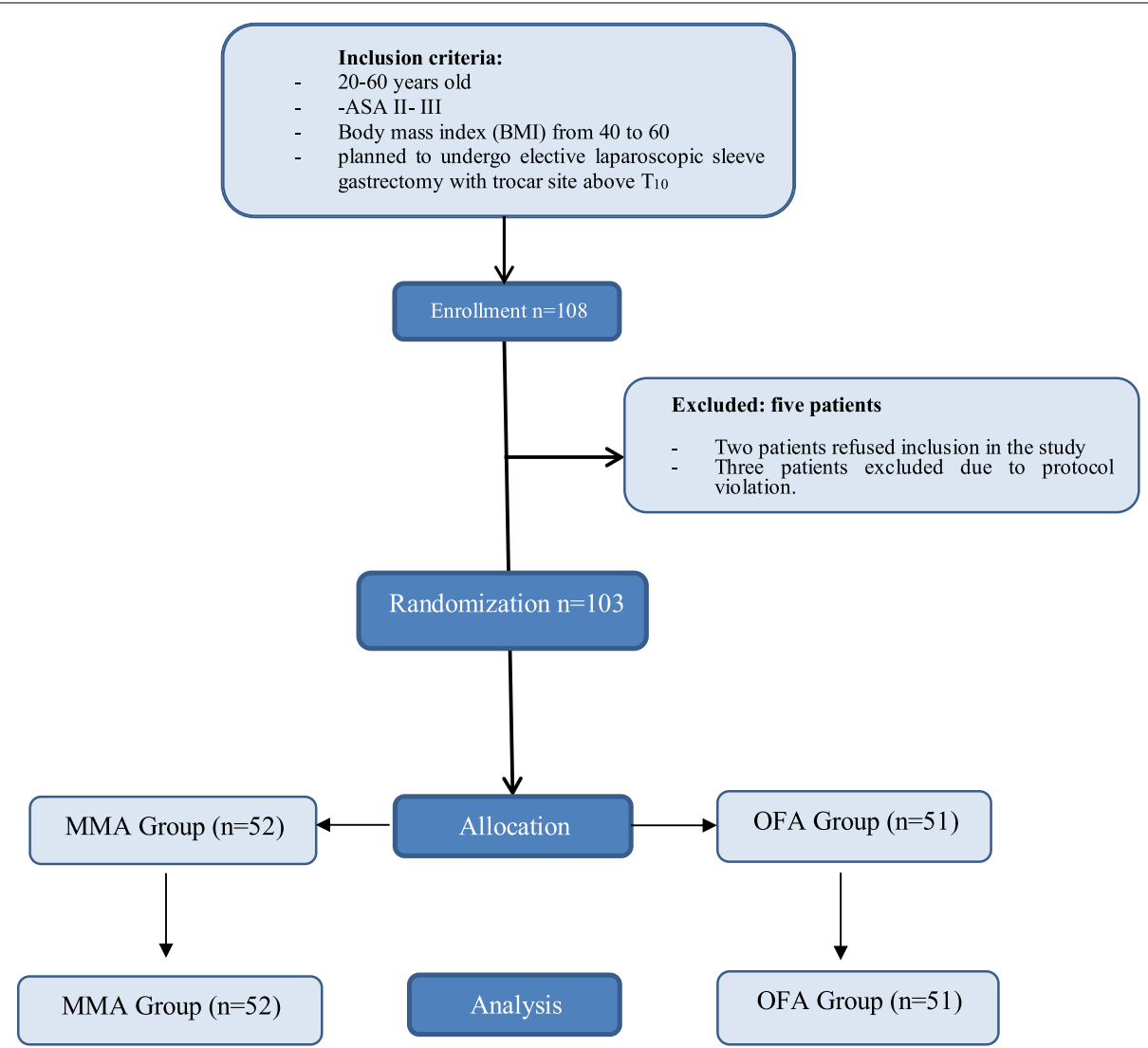

Fig. 1 Study Flow chart showing inclusion, enrollment, randomization, allocation, and analysis

A team of a single anaesthesiologist and surgeon standardized the preoperative workup, patient preparation; and arranged appropriate referrals to medical services for comorbidity optimization. Patients received preoperative teaching on how to rate his pain on defined numerical rating scale (NRS) with $0=$ no pain and 10 is the worst imaginable pain at the specified time verbally or written. All operations were performed using a standardized technique. An investigator blinded to both study groups was trained to interview patients and fill the quality of recovery 40 questionnaires (QoR-40) at 6 and $24 \mathrm{~h}$ postoperatively.

\section{Enhanced recovery and anaesthesia protocol}

A dedicated bariatric coordinator provided preoperative teaching to patients and caregivers about the QoR40 questionnaire and total plan of care: Preoperative oral fluid intake was encouraged, and patients were instructed to drink a $100 \mathrm{mg}$ carbohydrate $(\mathrm{CHO})$ load at midnight, followed by a $50 \mathrm{mg} \mathrm{CHO}$ load $2 \mathrm{~h}$ before surgery. Patients were premedicated with intravenous (IV) midazolam $25 \mu \mathrm{g} . \mathrm{kg}^{-1}$ in the preoperative holding area. Normothermia was maintained, and sequential pneumatic compression was applied before the start of surgery.

Following maximal pre-oxygenation (end-tidal oxygen $>90 \%$ ), general anaesthesia was induced using IV propofol $2 \mathrm{mg} . \mathrm{kg}^{-1}$ and fentanyl $1 \mu \mathrm{g}$. $\mathrm{kg}^{-1}$ in the MMA group while patients in the OFA group were premedicated with IV dexmedetomidine $0.1 \mu \mathrm{g} . \mathrm{kg}^{-1}$ in $100 \mathrm{ml}$ normal saline over $10 \mathrm{~min}$ then induced with propofol $\left(2 \mathrm{mg} . \mathrm{kg}^{-1}\right)$ -ketamine $\left(0.5 \mathrm{mg} . \mathrm{kg}^{-1}\right)$ mixture and maintained on dexmedetomidine $0.5 \mu \mathrm{g} . \mathrm{kg}^{-1} \cdot \mathrm{h}^{-1}$, ketamine $0.5 \mathrm{mg} \cdot \mathrm{kg}^{-1}$. $\mathrm{h}^{-1}$, and lidocaine $1 \mathrm{mg} . \mathrm{kg}^{-1} \cdot \mathrm{h}^{-1}$ were prepared in $50 \mathrm{ml}$ normal saline to run at a rate of $50 \mathrm{ml} \cdot \mathrm{h}^{-1}$. Cisatracurium $\left(0.15 \mathrm{mg} . \mathrm{kg}^{-1}\right)$ was used in both groups for muscle relaxation. Dosage was based on the ideal body weight using the Devine formula [16].

All Patients underwent endotracheal intubation and placement of a 36 French gastric calibration tube under video laryngoscopic guidance. Ultrasound-guided bilateral oblique subcostal transverse abdominis plane (OSTAP) block was performed in all patients using $40 \mathrm{ml}$ of $0.25 \%$ bupivacaine hydrochloride (Marcaine, Astra Zeneca UK) following intubation. Anaesthesia was 
maintained using Sevoflurane to 1.5-2.0 minimum alveolar concentration in air/oxygen with fractional inspired oxygen of 0.6, and a bispectral index (BIS) range between 40 and 60 in all patients. Standard ASA monitoring of patients included ECG, heart rate, pulse oximetry, noninvasive blood pressure, end tidal $\mathrm{Co} 2$ (Etco2) and core temperature.

The Ventilator was set to maintain normocapnia of $35-45 \mathrm{mmHg}$ and Spo2 between 94 and 100\%. All patients received parecoxib $40 \mathrm{mg} \mathrm{IV}$ after induction, and $1 \mathrm{~g}$ of paracetamol IV approximately $15 \mathrm{~min}$ prior to extubation. Furthermore, patients received dual intravenous antiemetic therapy; $8 \mathrm{mg}$ of ondansetron, and $8 \mathrm{mg}$ of dexamethasone.

Intraoperative hemodynamic parameters (MAP and heart rate) were recorded at 5-min intervals. Baseline values were taken $5 \mathrm{~min}$ after induction and a $15 \%$ rise in the MAP or HR prompted the administration of a $20 \mu \mathrm{g}$ fentanyl bolus in the MMA group versus a $10 \mathrm{mg}$ bolus of labetalol in the OFA group. Events number of MAP reduction (more than 20\% from baseline) and HR (less than $45 \mathrm{bpm}$ ) were recorded and managed by vasopressor or atropine respectively. Duration of surgery was defined as the time from the first incision to the completed wound dressing. After surgery, the reversal of neuromuscular blockade was administered to achieve a TOF of 0.9. Extubation time was defined as the time between the end of surgery and endotracheal extubation.

In the PACU, nurses blinded to the two groups administered $15 \mathrm{mg}$ of pethidine if the NRS was between 4 and 6 (moderate pain) versus $30 \mathrm{mg}$ if the NRS was greater than 7 (severe pain). The pain was assessed at 5-min intervals until pain relief was achieved (NRS $\leq 3$ ). Total pethidine dose in the PACU was recorded. The level of sedation was assessed $15 \mathrm{~min}$ after arrival to the PACU according to the Ramsay score [17]. Low saturation $(<94 \%)$, obstructed breathing, shivering or feeling cold, and duration of PACU stay were also recorded. Patients were discharged from the PACU if they achieved a modified Aldrete score of $\geq 9$ [18].

In the ward, all patients received $1 \mathrm{~g}$ of IV paracetamol 6 hourly, and $40 \mathrm{mg}$ IV parecoxib 12 hourly. Level of pain was measured using NRS on arrival to the ward at $0 \mathrm{~h}$, and at $2,4,6,12$, and $24 \mathrm{~h}$ postoperatively. An intravenous infusion of $25 \mathrm{mg}$ of pethidine over $15 \mathrm{~min}$ was administered if NRS $>3$, and the pain was re-assessed at $15 \mathrm{~min}$ intervals. An additional $10 \mathrm{mg}$ intravenous infusion of pethidine was given as needed till the NRS score dropped below 4. Four mg of Intravenous ondansetron and or $10 \mathrm{mg}$ of metoclopramide were administered to treat nausea or vomiting.

Patients were encouraged to ambulate, start oral ice chips, and void within $2 \mathrm{~h}$ from arrival to the ward. The timing of the first rescue analgesic dose or need for antiemetic therapy in the ward were recorded as well as further doses during the 24-h stay.

\section{Primary outcome}

A blinded investigator trained on the QoR-40 interviewed all patients and completed the form at 6 and $24 \mathrm{~h}$ from patient arrival to the ward. A transculturally validated arabic version of the questionnaire was used by the investigator in the interview. Twelve questions measured the comfort state; 9 questions measured the emotional state; 7 questions measured the psychological state; 5 questions measured the physical independence and 7 questions measured the level of pain. Each question received a score of 1 to 5 with a worst possible score of 40 and a best possible score of 200 [19].

\section{Secondary outcome}

Time to first independent ambulation, time to tolerate oral fluids, and time to readiness for discharge were measured according to the modified postoperative discharge scoring system (PADSS) [20]. The Blinded investigator-assessed patient readiness for discharge according to PADSS on an hourly basis and documented the time to achieve discharge eligibility in minutes. Patients were considered eligible for discharge if they achieved a total score $\geq 9$ on the condition that the vital signs parameter score was not less than 2 , and none of the other five parameters scored a zero.

Numerical rating scale (NRS) in the PACU, and on arrival to the ward at $0 \mathrm{~h}$ then at $2,4,6,12$, and $24 \mathrm{~h}$ postoperatively were recorded. Total pethidine consumption, calculated as an equivalent oral morphine dose, during the $24 \mathrm{~h}$, in the recovery unit (PACU) and at time of first rescue analgesic dose in the ward were recorded as well as the proportion of events with NRS $>4$.

Intraoperative hemodynamic parameters, surgical time, extubation time, PACU stay, and postoperative nausea and vomiting in the PACU, and in the ward were also documented.

\section{Statistical analysis}

Statistical analysis was done using IBM-SPSS 20 software (IBM Corp., Armonk, N.Y., USA). Prior published studies on heterogeneous patients undergoing cardiac, general, and neurosurgical procedures have suggested a $3.2 \%$ difference in the QoR- 40 score as clinically significant which we have used to calculate our sample size [21]. Adoption of ERAS in MMA and the use of OSTAP block have already improved the quality of recovery in laparoscopic and bariatric surgery to above the 90th percentile in almost all patients. Nowadays, most of the work focuses on improvement in the remaining $10 \%$ of 
the score. A difference of $1.5 \%$ in the score will translate to a $15 \%$ improvement in the non-achieved score and would probably be clinically significant if it results in change in the time to discharge, a target of ERAS. A sample size of 43 patients in each arm had a $90 \%$ power to detect the $3.2 \%$ difference in the mean score with a $3.5 \%$ standard deviation at an alpha of 0.05 using a two-sided two-sample equal-variance t-test. Fifty-four patients were recruited in each arm assuming a $20 \%$ drop-out rate.

The normality of the distribution of each of the continuous data was examined by the Kolmogorov-Smirnov test and quantile-quantile plot. Normally distributed continuous variables were presented as mean $\pm S D$; and were univariately compared using a two-sample Student's $t$ test. When continuous data were not normally distributed: the median, the interquartile range were presented and were compared using the Mann-Whitney test. The frequency or percentage was used to describe categorical data, and Fisher exact was the preferred test to compare the two groups whenever appropriate followed by the Pearson's chi-square test.

Number of events (number of events over total number of measurements) was used to analyze reduction in MAP (more than 20\% reduction from baseline), HR (less than $45 \mathrm{bpm}$ ) and NRS (more than 3 ) repeated measurements.

\section{Results}

One hundred and eight patients were allocated to both groups. Two patients refused inclusion in the study; three patients were excluded due to protocol violation (two patients received an out of protocol different postoperative analgesia by a new on-call physician unaware of the study protocol, and the third patient received sedation in the PACU due to severe agitation. One hundred and three patients were randomly allocated to the MMA or OFA groups. Patient characteristics were similar between the two groups (Table 1).

\section{Quality of recovery}

All patients answered the QoR-40 questionnaire without difficulty. The estimated difference in mean was used to compare the QoR-40 scores in the pre-study protocol. The total estimated difference in the mean QoR-40 score was $-4.15,95 \% \mathrm{CI}(-5.78$ to -2.5$)$ with a $P$ value $<0.0001$ at $6 \mathrm{~h}$; and was $0.738,95 \% \mathrm{CI}(-0.8$ to 2.28$)$, with a $P$ value $=0.345$ at $24 \mathrm{~h}$. However, we discovered later that the QoR-40 score followed a non- Gausian distribution.
Table 1 Clinical characteristics. Mean $\pm S D$, standard deviation; $M$, male: F, female; BMI, body mass index; IQR, Interquartile range, Times are shown in minutes

\begin{tabular}{|c|c|c|c|}
\hline & $\begin{array}{l}\text { Group I (MMA) } \\
(N=52)\end{array}$ & $\begin{array}{l}\text { Group II (OFA) } \\
(N=51)\end{array}$ & $P$ value\# \\
\hline Age $(y r .)^{a}$ & $32.0[23.0-39.0]$ & $30.0[22.0-36.0]$ & 0.242 \\
\hline $\operatorname{Sex}(M / F)^{b}$ & $21 / 31$ & $19 / 32$ & 0.745 \\
\hline ASA II & 35 & 29 & 0.275 \\
\hline ASA III & 17 & 22 & \\
\hline BMI $\left(\mathbf{k g} / \mathbf{m}^{2}\right)^{\mathrm{a}}$ & $44.0[42.0-45.0]$ & $45.0[43.0-46.0]$ & 0.062 \\
\hline $\begin{array}{l}\text { Duration of surgery } \\
(\min )^{c}\end{array}$ & $43.0 \pm 9.86$ & $40.5 \pm 9.04$ & 0.172 \\
\hline $\begin{array}{l}\text { Duration of anaesthesia } \\
(\min )^{c}\end{array}$ & $64.8 \pm 9.19$ & $63.7 \pm 8.61$ & 0.524 \\
\hline
\end{tabular}

-Values are given as mean \pm standard deviation and median [IQR]

$\# P$ value $<0.05$ significant

a Mann-Whitney test

${ }^{\mathrm{b}}$ Fisher's exact test

'Student's t-test

Table 2 The dimensions of the postoperative QoR-40 at 6 and 24 hours

\begin{tabular}{|c|c|c|c|}
\hline & $\begin{array}{l}\text { Group I (MMA) } \\
(N=52)\end{array}$ & $\begin{array}{l}\text { Group II (OFA) } \\
(N=51)\end{array}$ & $P$ value\# \\
\hline \multicolumn{4}{|l|}{ QoR-40 at $6 \mathrm{~h}$} \\
\hline Comfort (60) & $52.0[44.0-58.0]$ & $53.0[45.0-58.0]$ & $0.004^{\#}$ \\
\hline Emotion (45) & $42.0[40.0-51.0]$ & $44.0[36.0-45.0]$ & $<0.0001^{\#}$ \\
\hline $\begin{array}{l}\text { Physical independ- } \\
\text { ence (25) }\end{array}$ & $23.0[22.0-25.0]$ & $23.0[22.0-23.0]$ & $0.004^{\#}$ \\
\hline $\begin{array}{l}\text { Psychological sup- } \\
\text { port (35) }\end{array}$ & $34.0[33.0-35.0]$ & $34.0[33.0-35.0]$ & $0.016^{\#}$ \\
\hline Pain (35) & 30.0 [28.0-33.0] & $32.0[28.0-32.0]$ & $<0.0001^{\#}$ \\
\hline Total score $(200)^{*}$ & 180 [173-195] & 185 [173-191] & $<0.0001^{\#}$ \\
\hline \multicolumn{4}{|l|}{ QoR-40 at $24 \mathrm{~h}$} \\
\hline Comfort (60) & $56.0[50.0-60.0]$ & $56.0[48.0-60.0]$ & 0.198 \\
\hline Emotion (45) & $44.0[41.0-45.0]$ & $44.0[37.0-45.0]$ & 0.810 \\
\hline $\begin{array}{l}\text { Physical independ- } \\
\text { ence (25) }\end{array}$ & $24.0[23.0-25.0]$ & $24.0[21.0-24.0]$ & 0.310 \\
\hline $\begin{array}{l}\text { Psychological sup- } \\
\text { port (35) }\end{array}$ & $35.0[34.0-35.0]$ & $35.0[33.0-35.0]$ & 0.275 \\
\hline Pain (35) & $32.0[29.0-35.0]$ & $32.0[30.0-34.0]$ & 0.534 \\
\hline Total score (200) & 191 [181-198] & 191 [178-198] & 0.479 \\
\hline
\end{tabular}

The values are the median [Range]

Analysis by Mann-Whitney's test

$\# P$ value $<0.025$ significant

Hence, we used the Mann-Whitney's test to compare the median difference in the QoR-40 scores (Table 2). Both parametric and non-parametric tests gave the same level of significance. 
Significance was preserved after correcting the type-I error by the Bonferroni's correction for two measurements (significance threshold at 0.025)".

\section{Readiness for discharge}

The OFA group tolerated oral fluid earlier and quicker readiness for discharge than MMA group, $P$-value $=0.022$ and 0.001 respectively but did not influence time to ambulate $(p=0.169)$ (Table 3$)$.

\section{Pain assessment}

The median and interquartile range for the NRS scores in the OFA group was statistically lower than MMA group at PACU, and at 2 and $6 \mathrm{~h} \mathrm{(Fig.} \mathrm{2).} \mathrm{Furthermore,} \mathrm{the} \mathrm{pro-}$ portion of events median value with NRS $>3$ was 0.50 in the OFA group vs. 0.58 in the MMA group, $P=0.008$ (Table 3).

\section{Opioid consumption}

The parameters of opioid consumption (in PACU and total 24-h) were lower in the OFA group $P=0.005$ and 0.024 respectively (Table 3 ).

In the MMA group, sixteen (30.7\%) patients required rescue fentanyl for an MAP elevation with a mean dose of $11.83 \pm 20.79 \mu \mathrm{g}$; Eight patients $(15.3 \%)$ required a single rescue dose; Seven patients $(13.5 \%)$ required two doses; and only one patient required a third rescue dose. The median value of MAP reduction events was 0.28 in the OFA group vs. 0.1 in the MMA group, $P=0.002$. Only 3 patients in OFA group had severe bradycardia (HR less than $45 \mathrm{bpm}$ ) and treated promptly with atropine.
None of the patients in the OFA group needed labetalol for an elevation in the HR or MAP. Hemodynamics (HR and MAP) changes during surgery are shown in Fig. 3. Extubation time was $6.85 \pm 3.81 \mathrm{~min}$ in the MMA group versus $9.24 \pm 3.29 \mathrm{~min}$ in the OFA group, $P=0.001$.

Eighteen patients $(35.3 \%)$ in the OFA group experienced postoperative nausea or vomiting (PONV) versus $24(46.2 \%)$ in the MMA group, $P=0.262$. Nine patients (17.6\%)in the OFA group had hallucinations versus none in the MMA group. There were no observed adverse events related to the OSTAP block, and no symptoms or signs of systemic toxicity were associated with the use of local anesthetic agents.

\section{Discussion}

In our study, OFA enhanced the total quality of recovery score and the individual scores of its five dimensions at $6 \mathrm{~h}$ when compared to MMA using the validated QoR-40 questionnaire. Such enhancement disappeared at $24 \mathrm{~h}$. In essence, our study showed MMA had slower recovery when compared to OFA making the latter more suited for ERAS. OSTAP block under ERAS resulted in all patients achieving the 90th percentile. A change in the remaining 10 percentile is what any study should target within ERAS. Our study showed a $2.1 \%$ absolute difference which translates to a $21 \%$ improvement in the remaining 10 percentiles which is considered clinically relevant because it resulted in early tolerance of oral fluid intake and expedited discharge in the OFA group. Mulier et al. [22] in a randomized controlled trial conducted on 45 patients found the QoR-40 to be better in the opioid-free

Table 3 Comparison of analgesic efficacy and recovery criteria between both groups

\begin{tabular}{|c|c|c|c|}
\hline & $\begin{array}{l}\text { Group I (MMA) } \\
(N=52)\end{array}$ & $\begin{array}{l}\text { Group II (OFA) } \\
(N=51)\end{array}$ & $P$ value \\
\hline PACU morphine analgesia (mg) & $10[10-20]$ & $10[0-10]$ & 0.024 \\
\hline Total postoperative morphine consumption (mg) [PACU and Ward] & $20[10-30]$ & $10[10-20]$ & 0.005 \\
\hline Number (\%) of patients did not receive opioids in PACU & $10(19.2)$ & $18(35.3)$ & 0.068 \\
\hline Number (\%) of patients did not receive opioids in ward & $17(32.7)$ & $24(47.1)$ & 0.136 \\
\hline Number (\%) of patients did not receive opioids in PACU or ward & $7(13.5)$ & $7(13.7)$ & 0.969 \\
\hline Median proportion of events NRS > 3 & $0.58[0.41-0.66]$ & $0.50[0.16-0.66]$ & 0.008 \\
\hline Extubation time (min) & $5.50[4.0-9.5]$ & $9.0[9.0-11.0]$ & 0.0002 \\
\hline PACU stay (min) & $20.0[20.0-25.0]$ & $30.0[25.0-35.0]$ & $<0.0001$ \\
\hline Time of first rescue analgesia & $120.0[69.0-152.0]$ & $228.0[122.5-229.0]$ & $<0.0001$ \\
\hline Ramsay Sedation score in the PACU & $3.0[3.0-3.0]$ & $4.0[3.0-4.0]$ & $<0.0001$ \\
\hline Time to ambulate (min) & $169[122.5-216.5]$ & $150[107.5-195.5]$ & 0.190 \\
\hline Time to oral fluid tolerance (min) & $238[150.5-346.0]$ & $175[97.5-274.5]$ & 0.022 \\
\hline Time to readiness for home discharge (min) & $504.5[439-625.5]$ & $444.0[356-529]$ & 0.0009 \\
\hline
\end{tabular}

Analysis between groups was done using Mann-Whitney test

Values are given as median, $[\mathrm{IQR}]-P$ value $<0.05$ is considered significant 


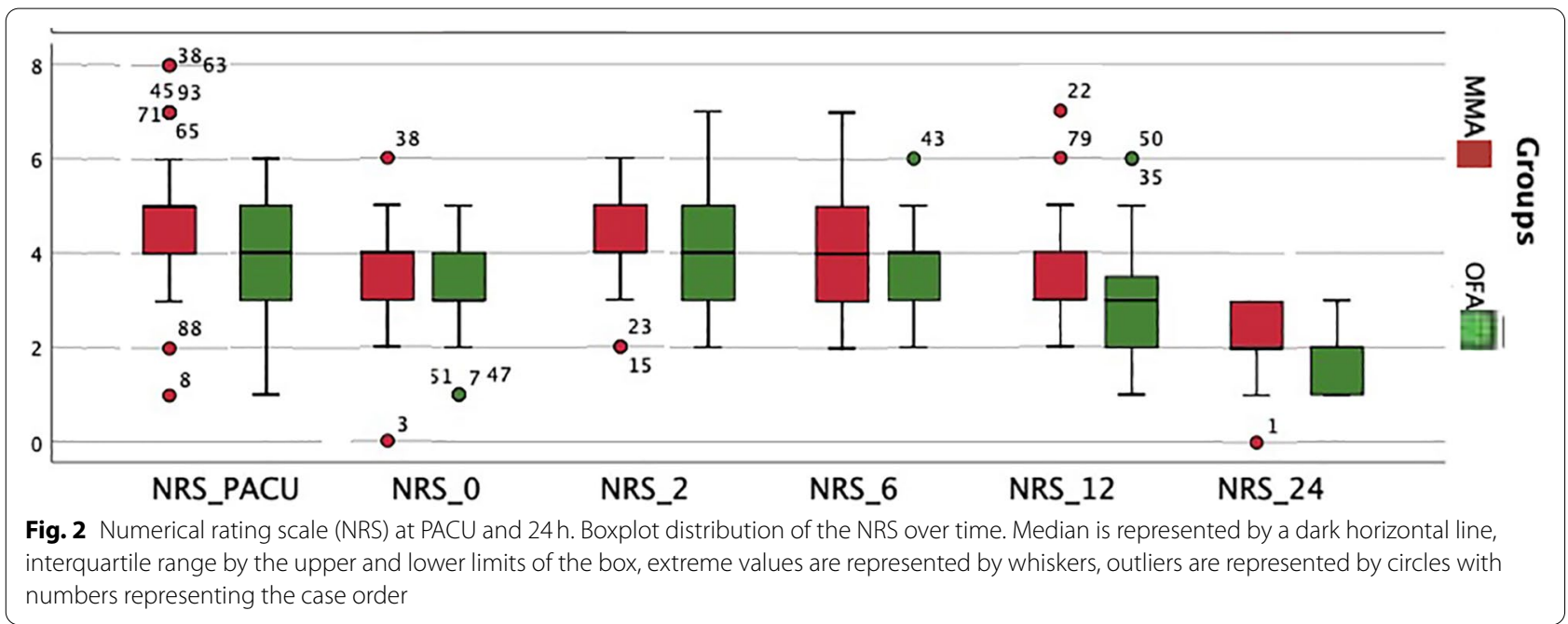

anaesthesia when compared to the opioid anaesthesia even at $24 \mathrm{~h}(p<0.001)$. We feel the use of a loco-regional block in our study and lack of maintenance opioid infusion improved the score in our MMA group by $27 \%$ over the opioid group in the Mulier study, and hence reduced the difference between the two groups in our study to a non-significant level at $24 \mathrm{~h}$.

Most studies have compared full opioid versus opioid-free anaesthesia [23-28]. Total opioid anaesthesia is falling out of favour and is rarely practiced since it is unanimously agreed that reducing opioid is beneficial. No studies have compared reduced opioid under multimodal analgesia versus opioid-free anaesthesia combined with loco-regional anaesthesia.

The low intraoperative opioid dose in our MMA group and in both groups postoperatively were deliberate. We tested OFA in a non-conventional way, as the control group had few intraoperative opioids, as all patients were covered by the intraoperative TAP block.

In a meta-analysis, Fletcher and Martinez showed that intraoperative administration of large doses of opioids caused increased pain perception, and postoperative opioid consumption [29]. The opioid paradox or opioid hyperalgesia is a state in which increased opioid dosage increases pain receptor sensitization, especially following short-acting opioids [13, 30]. Furthermore, opioid tolerance plays a role in increasing postoperative demand [31-33].

The improved score in the pain dimension of the QoR40 at $6 \mathrm{~h}$ in the OFA group was further supported by a reduced mean NRS score at $6 \mathrm{~h}$ in the same group. The combination of Dexmedetomidine [8], lidocaine [9], and ketamine [12] reduced the number of events with an NRS score $>4$; resulting in reduced opioid consumption in the PACU and ward. Even though fewer patients in the OFA group needed opioid analgesia in the PACU or ward, the difference failed to reach statistical significance (Fig. 2).

A retrospective study showed positive correlation between postoperative morphine consumption and the pain score, and just implementing ERAS resulted in a $41.8 \%$ reduction in the morphine equivalent dose, $P<0.001$ [34].

Zeltsman and colleagues [13] found more opioid consumption in the OFA $(23.25 \mathrm{mg})$ vs. $9.79 \mathrm{mg}$ in OA, $P=0.03$; a longer time $255.3 \mathrm{~min}$ to achieve an Aldrete score $>9$ in OFA versus $135.42 \mathrm{~min}$ in $\mathrm{OA}, P=0.03$; and $70 \%$ of OFA patients needed antiemetic rescue doses vs. $25 \%$ of OA patients, $P=0.08$. It is noteworthy that the pain score in both groups and PACU stay was much higher when compared to other studies in the literature. Though the authors cautioned against drawing a conclusion due to the small sample size and the possibility of random error, their study highlights the importance of not adopting OFA outside of an ERAS protocol.

Ziemann-Gimmel et al. [23] showed no difference in postoperative opioid consumption between OFA and OA. Yet, the OFA group had a statistically significant reduction in nausea $(p=0.02)$. The use of a lower ketamine dose $\left(0.5 \mathrm{mg} \cdot \mathrm{kg}^{-1}\right)$ at induction, as well as not having any form of regional block may explain the lack of difference in opioid consumption. The reduction in nausea in their OFA group was similar to what we have seen though it did not reach significance in our study. Furthermore, the significant absolute reduction in the proportion of patients suffering from nausea compared to many studies including ours might be explained by the preemptive use of a scopolamine transdermal patch plus triple prophylaxes with dexamethasone, ondansetron, and rescue doses of droperidol or promethazine in the PACU. 


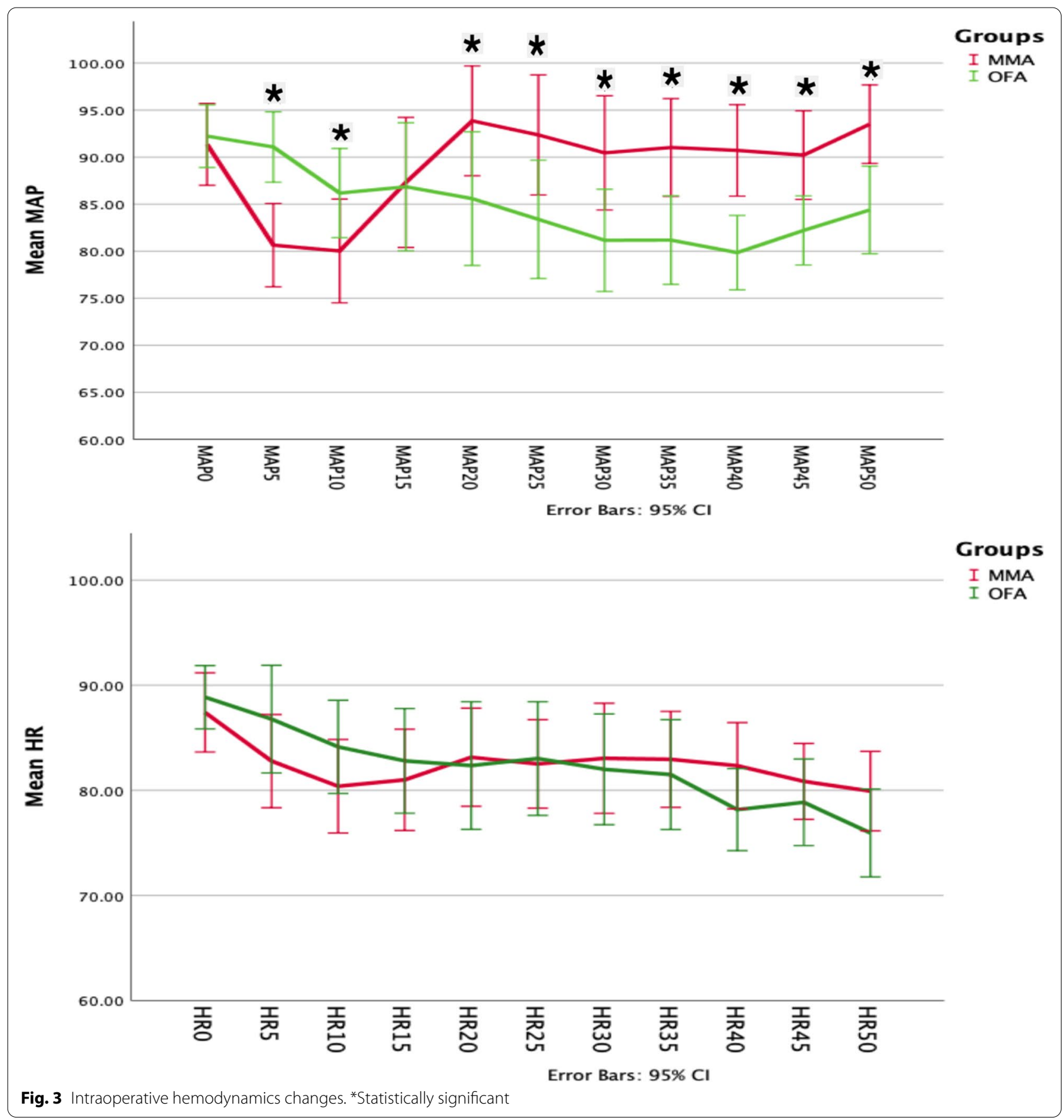

Several trials implementing the ERAS pathway in bariatric surgery concluded its efficacy in reducing the length of hospital stay, peri-operative opioid consumption, readmission rate, and incidence of PONV [35-40]. Patients in the OFA group were able to tolerate fluids earlier, had less nausea or vomiting, and potentially reduced their stay by $18 \%$, a desired outcome of ERAS in the era of managed care and capitation.
Use of Ketamine in short duration surgery in our patients may explain the prolonged extubation time, longer duration, and higher sedation score in the PACU for the OFA patients but it had no clinical relevance since the PACU stay was much shorter than most reported studies in the literature. There were 9 cases of mild hallucination (17.6\%) in the OFA group, a side effect reported in one trial using nearly the same ketamine dose [23]. 
Hallucinations were short-lived, self-aborted in the PACU, and were not remembered in the ward. Hemodynamics stability (MAP) was better under OFA and none of our patients experienced hypoxemia in the PACU.

Finally, this is the first high powered randomized trial that shows OFA can be more suited for ERAS in bariatric surgery.

We realized from a pilot study performed on ten patients that the first $6 \mathrm{~h}$ were more relevant in sleeve gastrectomy done under ERAS so we decided to add a measurement of the quality of recovery at $6 \mathrm{~h}$ which proved later to be valuable. Inability to compare the length of stay between both groups is one limitation of the study since the health authority in the country forbids same-day discharge in bariatric surgery. We believe our study will encourage change to this policy. To overcome this limitation, we measured the time to readiness for discharge based on PADSS as a substitute. Implementing ERAS has been proven to reduce hospital stay [32]. Our study showed adding OFA expedited readiness for discharge by an absolute $22 \%$ in addition to the improvement in the quality of recovery as discussed earlier.

A second limitation or challenge occurred during blinding and provision of the intervention by the principal investigator. That was overcome by having an independent second investigator collect and analyze the data independently.

\section{Conclusion}

Opioid free anaesthesia when combined with Locoregional anaesthesia is better suited to satisfy the goals of ERAS. It provides early improvement of the quality of recovery; improves postoperative pain; and reduces opioid consumption over multimodal analgesia. A larger multi-centre randomized controlled trial is needed to standardize the optimal anaesthetic and surgical considerations in ERAS in bariatric surgery.

\begin{abstract}
Abbreviations
ASA: American Society of Anesthesiologists; BIS: Bispectral index; BMI: Body Mass Index; ERAS: Enhanced Recovery after Surgery; Etco2: End tidal Co2; IV: Intravenous; IQR: Interquartile range; MAP: Mean arterial pressure; MMA: Multimodal analgesia; NMDA: N-methyl d aspartate; NSAID: Non-steroidal antiinflammatory drugs; NRS: Numerical rating scale; OFA: Opioid-free anaesthsia; OSTAP: Oblique subcostal transverse abdominis plane; PACU: Post-anaesthesia care unit; PADSS: Postoperative discharge scoring system; PONV: Postoperative Nausea and Vomiting; QOR-40: Quality of Recovery Score- 40; TOF: Train of four.
\end{abstract}

\section{Acknowledgements}

1. Assistance with the article: We are immensely grateful to Dr. Ahed Zidan, Anesthesiology consultant and scientist at King Fahd Specialist Hospital, Dammam, KSA. Dr. Ahed contributed valuable input on study design and provided independent review and critique of the manuscript. Our gratitude to the surgical team, operating theatre, recovery and inpatient nurses of Al Mashfa Medical Center for their endless support and contribution.

\section{Authors' contributions}

MI made 1-Concept, design, the definition of intellectual content. 2- Literature search. 3- Data acquisition and analysis. 4- Statistical analysis. 5- Manuscript preparation, editing, and review. 6- Integrity of the work as a whole from inception to published article. AE made the: 1-Concept, design 2- Literature search. 3- Data acquisition and analysis. 4- Manuscript preparation. 5- Final approval of the version to be published. 6- Agreement on all aspects of the work. AH made the: 1-Concept, design 2- Literature search. 3- Manuscript preparation. 4- Final approval of the version to be published. 5- Agreement on all aspects of the work. OA made the 1- Data acquisition and analysis. 2- Manuscript preparation. 3- Final approval of the version to be published. 4- Agreement on all aspects of the work. OE made the 1-Concept, design, the definition of intellectual content. 2- Literature search. 3- Data acquisition and analysis. 4- Statistical analysis. 5- Manuscript preparation, editing, and review. 6- Integrity of the work as a whole from inception to published article. All authors have read and approved the manuscript.

\section{Funding}

None.

\section{Availability of data and materials}

The data set used and/or analyzed during the current study are submitted in a separate file.

Data can be made available from the corresponding author upon request at any stage.

\section{Declarations}

Ethics approval and consent to participate

The study was conducted at Almashfa Medical Center in Alkhobar Saudi Arabia between March and August 2020 (ClinicalTrials.gov: NCT04285255; registered Study Chair: Mohamed Ibrahim; registration date: February 2020). Approval was provided by the Almashfa ethics committee (Chairperson Dr. Mohamed Ramadan) on January 20, 2020, under the number 1/1-2020. Written informed consents were obtained from patients or their legal representatives before surgery. All methods were performed in accordance with the relevant guidelines and regulations. The trial was registered before patient enrollment.

\section{Consent for publication \\ Not applicable.}

\section{Competing interests}

We declare that there is no conflict of interest.

\section{Author details}

${ }^{1}$ Department of Anesthesiology, Faculty of Medicine, Zagazig University, Zagazig, Egypt. ${ }^{2}$ Al Mashfa Medical Center, Arraka Shamalia Dist., Khalid Ibn Elwaleed Street, Al Khobar, Kingdom of Saudi Arabia.

Received: 25 May 2021 Accepted: 28 December 2021 Published online: 21 January 2022

\section{References}

1. Macfater H, Xia W, Srinivasa S, et al. Evidence-based Management of postoperative pain in adults undergoing laparoscopic sleeve gastrectomy. World J Surg. 2019;43(6):1571-80.

2. Ruiz-Tovar J, Muñoz JL, Gonzalez J, et al. Postoperative pain after laparoscopic sleeve gastrectomy: Comparison of three analgesic schemes (isolated intravenous analgesia, epidural analgesia associated with intravenous analgesia and port-sites infiltration with bupivacaine associated with intravenous analgesia). Surg Endosc. 2017;31(1):231-6.

3. Soffin EM, Wetmore DS, Beckman JD, et al. Opioid-free anaesthesia within an enhanced recovery after surgery pathway for minimally invasive lumbar spine surgery: A retrospective matched cohort study. Neurosurg Focus. 2019;46(4):E8.

4. Brummett CM, Waljee JF, Goesling J, Moser S, Lin P, Englesbe MJ, et al. New persistent opioid use after minor and major surgical procedures in 
US adults. JAMA Surgery. 2017;152(6):e170504. https://doi.org/10.1001/ jamasurg.2017.0504.

5. Mulier JP. Perioperative opioids aggravate obstructive breathing in sleep apnea syndrome: mechanisms and alternative anaesthesia strategies. Curr Opin Anaesthesiol. 2016;29(1):129-33. https://doi.org/10.1097/ACO. 0000000000000281

6. Ibrahim M, El Shamaa H. Efficacy of ultrasound-guided oblique subcostal transversus abdominis plane block after laparoscopic sleeve gastrectomy: a double-blind, randomized, placebo-controlled study. Egypt J Anaesth. 2014;30:285-92

7. Samuels D, Abou-Samra A, Dalvi P, Mangar D, Camporesi E. Opioid-free Anaesthesia results in reduced postoperative opioid consumption. J Clin Anesth Pain Med. 2017;2(1):1-3.

8. Gaszynski T, Czarnik K, Lasziniski L, Gaszynski W. Dexmedetomidine for attenuating hemodynamic response to intubation stimuli in morbidly obese patients anesthetized using the low-opioid technique: comparison with fentanyl-based general anaesthesia. Anaesthesiol Intensive Ther. 2016;48(5):275-9. https://doi.org/10.5603/AlT.a2016.0058.

9. De Oliveira GS Jr, Duncan K, Fitzgerald P, Nader A, Gould RW, McCarthy RJ. Systemic lidocaine to improve the quality of recovery after laparoscopic bariatric surgery: a randomized double-blinded placebo-controlled trial. Obes Surg. 2014;24(2):212-8. https://doi.org/10.1007/s11695-013-1077-x.

10. Ventham NT, Kennedy ED, Brady RR, et al. Efficacy of intravenous lidocaine for postoperative analgesia following laparoscopic surgery: a meta-analysis. World J Surg. 2015;39(9):2220-34. https://doi.org/10.1007/ s00268-015-3105-6.

11. Kumar K, Kirksey MA, Duong S, Wu CL. A review of opioid-sparing modalities in perioperative pain management: methods to decrease opioid use postoperatively. Anesth Analg. 2017;125:1749-60.

12. Chan JW, Shetty P. Does the use of ketamine or magnesium decrease postoperative pain scores in laparoscopic bariatric surgery for morbid obesity? Anesth Analg. 2016;123(3):814-5. https://doi.org/10.1213/01. ane.0000493012.08816.5b.

13. Zeltsman M., Aronsohn J, Gerasimov M, Palleschi G. Does Opioid-free anaesthesia Make a Difference in Bariatric Surgery? In: American society of anesthesiologists annual meeting on October 22, 2019. Orlando.

14. Feld JM, Laurito CE, Beckerman M, Vincent J, Hoffman WE. Non-opioid analgesia improves pain relief and decreases sedation after gastric bypass surgery. Can J Anaesth. 2003;50(4):336-41. https://doi.org/10.1007/BF030 21029.

15. Myles PS, Weitkamp B, Jones K, Melick J, Hensen S. Validity and reliability of a postoperative quality of recovery score: the QoR-40. Br J Anaesth. 2000;84(1):11-5. https://doi.org/10.1093/oxfordjournals.bja.a013366.

16. Pai MP, Paloucek FP. The origin of the "ideal" body weight equations. Ann Pharmacother. 2000;34(9):1066-9.

17. Ramsay MA, Savage TM, Simpson BR, Goodwin R. Controlled sedation with alfaxalone-alphadolone. BMJ. 1974;2:656-9.

18. Aldrete JA. Modifications to the post-anaesthesia score for use in ambulatory surgery. J Perianesth Nurs. 1998;13(3):148-55. https://doi.org/10. 1016/s1089-9472(98)80044-0

19. Terkawi AS, Myles PS, Riad W, et al. Development and validation of Arabic version of the postoperative quality of recovery-40 questionnaire. Saudi J Anaesth. 2017;11(Suppl 1):S40-52. https://doi.org/10.4103/sja.SJA_77_17.

20. Palumbo P, Tellan G, Perotti B, Pacilè MA, Vietri F, Illuminati G. Modified PADSS (post Anaesthetic discharge scoring system) for monitoring outpatients discharge. Ann Ital Chir. 2013;84(6):661-5.

21. Myles PS, Myles DB, Galagher W, Chew C, MacDonald N, Dennis A. Minimal clinically important difference for three quality of recovery scales. Anesthesiology. 2016;125(1):39-45. https://doi.org/10.1097/ALN.00000 00000001158

22. Mulier JP, Wouters R, Dillemans B, De Kock M. A randomized controlled, double-blind trial evaluating the effect of opioid-free versus opioid general anaesthesia on postoperative pain and discomfort measured by the QoR-40. J Clin Anesth Pain Med. 2018;2:015.

23. Ziemann-Gimmel P, Goldfarb AA, Koppman J, Marema RT. Opioidfree total intravenous anaesthesia reduces postoperative nausea and vomiting in bariatric surgery beyond triple prophylaxis. $\mathrm{Br} J$ Anaesth. 2014;112(5):906-11. https://doi.org/10.1093/bja/aet551.

24. Beloeil H. Opioid-free anaesthesia. Best Pract Res Clin Anaesthesiol. 2019;33(3):353-60. https://doi.org/10.1016/j.bpa.2019.09.002.
25. Bakan M, Umutoglu T, Topuz U, Uysal H, Bayram M, Kadioglu H, et al. Opioid-free total intravenous anaesthesia with propofol, dexmedetomidine and lidocaine infusions for laparoscopic cholecystectomy: a prospective, randomized, double-blinded study. Braz J Anesthesiol. 2015;65:191-9.

26. Gaszynski T, Czarnik K, Lasziniski L, Gaszynski W. Dexmedetomidine for attenuating hemodynamic response to intubation stimuli in morbidly obese patients anesthetized using the low-opioid technique: comparison with fentanyl-based general anaesthesia. Anaesthesiol Intensive Ther. 2016:48(5):275-9.

27. Lam K, Mui W. Multimodal analgesia model to achieve low postoperative opioid requirement following bariatric surgery. Hong Kong Med J. 2016;25(5):428-34

28. Mansour MA, Mahmoud AA, Geddawy M. Nonopioid versus opioidbased general anaesthesia technique for bariatric surgery: a randomized double-blind study. Saudi J Anaesth. 2013;7(4):387-91. https://doi.org/10. 4103/1658-354X.121045

29. Fletcher $D$, Martinez V. Opioid-induced hyperalgesia in patients after surgery: A systematic review and a meta-analysis. Br J Anaesth. 2014;112(6):991-1004. https://doi.org/10.1093/bja/aeu137.

30. Mulier J. Opioid free general anaesthesia : a paradigm shift? Anaesthesia libre de opioids: ¿un Cambio de paradigma? Rev Esp Anestesiol Reanim. 2017;64(8):427-30. https://doi.org/10.1016/j.redar.2017.03.004.

31. Rivat C, Ballantyne J. The dark side of opioids in pain management: basic science explains clinical observation. Pain Rep. 2016;1 (2):e570. https://doi. org/10.1097/PR9.0000000000000570. Published 2016 Sep 8

32. Forget P. Opioid-free anaesthesia. Why and how? A contextual analysis. Anaesth Crit Care Pain Med. 2019;38(2):169-72. https://doi.org/10.1016/j. accpm.2018.05.002

33. Lavand'homme P, Steyaert A. Opioid-free anaesthesia opioid side effects: tolerance and hyperalgesia. Best Pract Res Clin Anaesthesiol. 2017:31(4):487-98. https://doi.org/10.1016/j.bpa.2017.05.003.

34. Ma P, Lloyd A, McGrath M, et al. Reduction of opioid use after implementation of enhanced recovery after bariatric surgery (ERABS). Surg Endosc. 2020;34:2184-90.

35. Awad S, Carter S, Purkayastha $S$, et al. Enhanced recovery after bariatric surgery (ERABS): clinical outcomes from a tertiary referral bariatric center. Obes Surg. 2014;24(5):753-8. https://doi.org/10.1007/s1 1695-013-1151-4.

36. Blanchet MC, Gignoux B, Matussière Y, et al. Experience with an enhanced recovery after surgery (ERAS) program for bariatric surgery: comparison of MGB and LSG in 374 patients. Obes Surg. 2017;27(7):1896-900. https:// doi.org/10.1007/s11695-017-2694-6.

37. Singh PM, Panwar R, Borle A, et al. Efficiency and safety effects of applying ERAS protocols to bariatric surgery: a systematic review with Meta-analysis and trial sequential analysis of evidence. Obes Surg. 2017;27(2):489501. https://doi.org/10.1007/s11695-016-2442-3.

38. Sinha A, Jayaraman L, Punhani D, Chowbey P. Enhanced recovery after bariatric surgery in the severely obese, morbidly obese, super-morbidly obese and super-super morbidly obese using evidence-based clinical pathways: a comparative study. Obes Surg. 2017;27(3):560-8. https://doi. org/10.1007/s11695-016-2366-y.

39. Lemanu DP, Singh PP, Berridge $K$, et al. Randomized clinical trial of enhanced recovery versus standard care after laparoscopic sleeve gastrectomy. Br J Surg. 2013;100(4):482-9. https://doi.org/10.1002/bjs.9026.

40. Mannaerts GH, van Mil SR, Stepaniak PS, et al. Results of implementing an enhanced recovery after bariatric surgery (ERABS) protocol. Obes Surg. 2016;26(2):303-12. https://doi.org/10.1007/s11695-015-1742-3.

\section{Publisher's Note}

Springer Nature remains neutral with regard to jurisdictional claims in published maps and institutional affiliations. 